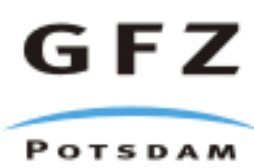

Originally published as:

Jahn, S., Martonak, R. (2008): Plastic deformation of orthoenstatite and the ortho- to highpressure clinoenstatite transition: a metadynamics simulation study. - Physics and Chemistry of Minerals, 35, 1, 17-23,

DOI: 10.1007/s00269-007-0194-2 


\title{
Plastic deformation of orthoenstatite and the ortho- to high-pressure clinoenstatite transition: A metadynamics simulation study
}

\author{
Sandro Jahn ${ }^{1, *}$ and Roman Martoňák ${ }^{2}$ \\ ${ }^{1}$ GeoForschungsZentrum Potsdam, Department 4, Telegrafenberg, 14473 Potsdam, \\ Germany \\ *Corresponding author \\ Tel.: +493312881483 \\ Fax: +493312881402 \\ E-mail: jahn@gfz-potsdam.de \\ ${ }^{2}$ Department of Experimental Physics, Faculty of Mathematics, Physics and \\ Informatics, Comenius University, Mlynská dolina F2, 84248 Bratislava, Slovakia
}

\begin{abstract}
Atomic-scale mechanisms of plastic deformation in orthoenstatite, $\mathrm{MgSiO}_{3}$ are studied by computer simulation methods. The combined use of metadynamics and molecular dynamics allows a direct observation of the structural changes during the creation of stacking faults in the (100) plane. A sequence of slip deformations in two different (100) planes at $P=15 \mathrm{GPa}$ and $T=1000 \mathrm{~K}$ reveals a probable transformation mechanism for the ortho- to high-pressure clinopyroxene transition. Each of the observed slips consist of at least four partial deformations crossing high energy intermediate structures. In agreement with experimental studies, both (100)[010] and (100)[001] slip systems are activated in the deformation process. The observation of a dominant (100)[001] single slip system in pyroxenes may be related to the fact that high energy intermediate dislocations with (100)[010] component are not stable on geological or experimental timescales.
\end{abstract}

Keywords: Molecular dynamics; Metadynamics; Phase transition; Enstatite; $\mathrm{MgSiO}_{3}$; Orthopyroxene; Clinopyroxene 


\section{Introduction}

Silicate pyroxenes, $\mathrm{M}_{2} \mathrm{Si}_{2} \mathrm{O}_{6}(\mathrm{M}=\mathrm{Na}, \mathrm{Li}, \mathrm{Mg}, \mathrm{Ca}, \mathrm{Mn}, \mathrm{Fe}, \mathrm{Ti}, \mathrm{Al})$, are important constituents of the Earth's crust and upper mantle. They are expected to have considerable influence on geophysical and rheological properties of the lithosphere and the asthenosphere. Depending on composition and thermodynamic conditions, pyroxenes may exist in a number of polymorphic crystal structures. Common to all pyroxenes is the close packing of oxygen in layers. The cations are arranged in alternating layers such that layers of corner-sharing tetrahedra are linked by edgesharing octahedra. Different polymorphic pyroxene structures are characterized by the sequence of close-packed layers and the distortion of the polyhedra (see e.g. Thompson and Downs (2003)).

Two of the most important polymorphs for the understanding of the Earth's interior are orthorhombic orthopyroxene with space group Pbca and monoclinic clinopyroxene with space group $C 2 / c$. In magnesium silicate, $\mathrm{MgSiO}_{3}$, these two phases are observed at high temperatures, $T\left(>700^{\circ} \mathrm{C}\right)$, and pressures, $P$, up to about $15 \mathrm{GPa}$. Orthoenstatite (OEn) is thermodynamically stable up to about 7 to $10 \mathrm{GPa}$, depending on $T$, whereas at higher pressures high-pressure clinoenstatite (high-P CEn) becomes the stable phase (Presnall, 1995). A similar phase behavior is observed in ferrosilite, $\mathrm{FeSiO}_{3}$, but the phase transition is shifted to somewhat lower pressure (Woodland and Angel, 1997). The ortho- to high-P clinopyroxene transition in the $(\mathrm{Mg}, \mathrm{Fe}) \mathrm{SiO}_{3}$ system leads to a volume change of about 3\% (Woodland and Angel, 1997). At mantle relevant composition $\left(x_{\mathrm{Fe}} \sim 0.1\right)$, the transition is expected to occur at about $300 \mathrm{~km}$ depth, where it may be responsible for a small magnitude seismic discontinuity, the "X-discontinuity" (Woodland, 1998).

A better understanding of the sharpness of a phase transition may be obtained if its nature and the atomistic transition mechanisms are known. Similarly, rheological properties of minerals, e.g. the ability of plastic deformation, are determined by nanoscale processes. The rather sluggish phase transition, especially at low $T$, between OEn and CEn suggests a reconstructive mechanism with a considerable kinetic barrier due to the required breaking of bonds. Recent measurements of elastic wave velocities showed that OEn can be substantially overpressurized to at least $P=15 \mathrm{GPa}$ at ambient 
$T$, which is close to the upper limit of the thermodynamic stability field of high-P CEn. The anomalous behavior of the wave velocities above $9 \mathrm{GPa}$ even suggests the formation of a metastable high-P OEn phase, which is likely to be related to OEn by a displacive phase transition (Kung et al., 2004; Jahn, 2007).

From a number of experimental studies of stacking faults in pyroxenes using transmission electron microscopy (TEM) it was concluded that the mechanism responsible for the transition from OEn to CEn is a slip parallel to the close packed oxygen layers in the (100) plane along the $\mathrm{SiO}_{4}$ chains, i.e. in [001] direction (Coe and Müller, 1973; Coe and Kirby, 1975; McLaren and Etheridge, 1976; Hugh-Jones et al., 1996). This picture is supported by the observation that the kinetics of the transition does not only depend on $P, T$ and the chemical composition but also on the presence of non-hydrostatic stress fields. The latter seem to help the activation of the partly reconstructive transition (Coe and Kirby, 1975).

Although TEM observations yield access to atomic structures, they do not provide direct access to the dynamics of the deformation or phase transition. The mechanism of atomic displacements is merely a matter of interpretation. Moreover, the high-P CEn phase is not quenchable to ambient conditions but transforms to the low-CEn phase (space group $P 2_{1} / c$ ). Hence the recovered samples for TEM investigations are necessarily altered, although small amounts of the high-P clinopyroxene phase persisted in quenched samples of ferrosilite (Hugh-Jones et al., 1996).

Aim of this study is to shed light on the transition mechanisms from OEn to high-P CEn using atomistic computer simulation methods. Molecular dynamics (MD) simulation is the method of choice to study displacive phase transitions. In the $\mathrm{MgSiO}_{3}$ system, this method was successfully employed to study, e.g., transitions between lowand high-P CEn (Miyake et al., 2002; Jahn and Madden, 2007) or between low- and high-T CEn (Shimobayashi et al., 2001). Reconstructive phase transitions, however, are often not accessible within MD time scales (picoseconds, ps, to nanoseconds, ns). If they are, considerable overpressurization may be needed as in the case of high-P phase transitions in $\mathrm{Al}_{2} \mathrm{O}_{3}$ (Jahn et al., 2004). We therefore applied the metadynamics-based approach (Martoňák et al., 2003, 2005, 2006) which was constructed to enable simulations of structural transitions involving crossing of large barriers. This technique was successful in simulation of complex reconstructive structural transformations in 
various silicate systems (Ceriani et al., 2004; Oganov et al., 2005; Martoňák et al., 2006, $2007 \mathrm{~b}, 2007 \mathrm{a}$ ) and is well suited also to our problem.

\section{Computational details}

The simulations are performed on enstatite supercells containing 640 atoms (384 O, 128 $\mathrm{Mg}, 128 \mathrm{Si}$ ) using periodic boundary conditions. For describing the interatomic forces in the MD simulations, an advanced ionic interaction potential, the Aspherical Ion Model (AIM) is used (Aguado et al., 2003; Madden et al., 2006). The model assumes an ionic system in which formally charged particles interact. It considers short-range repulsion between ions due to the overlap of the electronic orbitals and attractive dispersion interactions. In addition, it takes into account anion polarization effects and allows for asymmetric deformations of the anions. Recently, an AIM potential was parameterized for the $\mathrm{Ca}-\mathrm{Mg}-\mathrm{Al}-\mathrm{Si}-\mathrm{O}$ (CMAS) system using ab initio electronic structure calculations, i.e. without reference to experimental data (Jahn and Madden, 2007). This potential was shown to be accurate and transferable in a wide range of chemical compositions and thermodynamic conditions. The potential reproduces well the lattice parameters and elastic constants of various $\mathrm{MgSiO}_{3}$ polymorphs ranging from ambient to $P-T$ conditions of the core-mantle boundary. Furthermore, it predicts correctly the transition pressure of the low- to high-P CEn displacive phase transition (Jahn and Madden, 2007).

These results provide confidence to use this AIM potential in the present study. To ensure that it also gives a good representation of OEn and high-P CEn, lattice parameters, elastic constants and enthalpy curves of the two phases were calculated. In the athermal limit, the enthalpy curves of OEn and high-P CEn cross at about $P=5 \mathrm{GPa}$, which is in very good agreement with the experimental transition pressure extrapolated to $T=0, P \sim 4$ to $5 \mathrm{GPa}$ (Presnall, 1995). In Table 1, the lattice parameters and elastic constants of OEn predicted by the AIM are compared to experimental results at ambient conditions. Agreement is reasonable with less than two percent deviation in the lattice parameters and up to about $20 \%$ deviations in the elastic constants.

The initial OEn configuration for metadynamics is equilibrated in a MD simulation at $P=15 \mathrm{GPa}$ and $T=1000 \mathrm{~K}$ using a time step of $\Delta t=1 \mathrm{fs}$ and a total equilibration time of 20 ps. Temperature and pressure are controlled by a Nose-Hoover thermostat coupled to 
a barostat (Martyna et al., 1994). The transition pressure between OEn and high-P CEn increases to about $P=7 \mathrm{GPa}$ at $T=1000 \mathrm{~K}$ (Presnall, 1995). Hence, the chosen $\mathrm{P} / \mathrm{T}$ conditions for the metadynamics simulations ( $T=1000 \mathrm{~K}, P=15 \mathrm{GPa})$ are well within the stability field of the high-P CEn phase with the initial OEn existing as a metastable phase.

We applied the recent version of the metadynamics algorithm described in Martoňák et al. (2006, 2007a) which employs the components of the scaled box matrix as collective variables. In total, 1500 metadynamics steps were performed. The external pressure was fixed to $P=15 \mathrm{GPa}$. At each metadynamics step a short ( $1 \mathrm{ps}$ ) constant volume molecular dynamics run was performed in order to relax the atomic positions and calculate the average of stress tensor required by the metadynamics algorithm. In these MD runs the constant temperature of $T=1000 \mathrm{~K}$ was controlled by the thermostat. In the metadynamics, a Gaussian height of $8.32 \mathrm{eV}$ is used, which corresponds to $0.065 \mathrm{eV}$ per $\mathrm{MgSiO}_{3}$ unit. The corresponding Gaussian width is $2.88 \mathrm{eV}^{1 / 2}$. The scaled coordinates were defined using the Hessian matrix calculated for the initial configuration.

For analysis of the structures obtained, the final configurations of the metadynamics steps are relaxed in constant $\mathrm{P} / \mathrm{T}$ MD simulations for $2 \mathrm{ps}$ (each). The respective coupled thermostat/barostat is set to ambient temperature, $T=300 \mathrm{~K}$, to reduce thermal fluctuations retaining the high pressure $(P=15 \mathrm{GPa})$. Enthalpies and simulation cell parameters of the relaxed structures are obtained by averaging over the last quarter of the run, i.e. over a time period of $0.5 \mathrm{ps}$. Displacement vectors are calculated from representative Si ions in the different layers along [100] using the final configuration of the relaxed cells. Displacements that lead to an increase of simulation cell angle $\beta$ or to a decrease of simulation cell angle $\gamma$ are defined as positive.

\section{Results}

The evolution of simulation cell parameters and enthalpy are shown in figure 1 . In Figures 1a, c, and e, average values during metadynamics are shown, whereas Figures $1 \mathrm{~b}, \mathrm{~d}$, and $\mathrm{f}$ represent the respective data after relaxation to hydrostatic conditions ( $T=300 \mathrm{~K}, P=15 \mathrm{GPa}$ ). During metadynamics, the Gibbs free energy of the unrelaxed configurations is expected to increase as long as the same free energy basin is 
filled with Gaussians. Since we are working at a temperature which is less than half of the melting temperature of the system, we assume that for visualization of the evolution of the system enthalpy represents a qualitatively good approximation to the Gibbs free energy. A sudden drop in enthalpy means that a new local energy minimum is reached, likely related to some structural change. In Figure 1e, the most prominent abrupt enthalpy minima are observed after 388, 475, 900 and 1001 steps. The latter is artificial caused by a restart of metadynamics after 1000 steps with a relaxed configuration. Further, a slow enthalpy decrease is observed between metasteps 1200 and 1350. The same enthalpy drops (except that at 1001 steps) are also observed in the enthalpy curve of the relaxed configurations (Figure 1f). They are closely related to structural changes indicated by changes in the lattice parameters (Figures 1a-d).

In the following the structural changes will be analyzed using the relaxed configurations, where elastic strain has been removed. Projections of selected relaxed configurations along [010] and [001] are shown in figure 2. The initial simulation cell is a $1 \times 2 \times 4$ supercell of OEn. Up to metastep 326, the structure remains orthorhombic in the space group Pbca. At step 18, a more dense metastable orthorhombic structure is observed. This structure has a $c$-parameter, that is about 3.5\% smaller than that of OEn, whereas $a$ and $b$ increase by about $1 \%$. The resulting decrease in volume is slightly less than $2 \%$. This metastable phase is characterized by different rotation angles within the tetrahedral chains and transforms back to OEn via a displacive mechanism at high $T$ (Jahn, 2007). Small fluctuations in the simulation cell lengths of figure 1b, especially in $b$ and $c$, are caused by similar transitions between structurally similar configurations of different density.

A first stacking fault in the (100) plane of OEn is created in metastep 327 by a glide of about $[0,0.2,0]$. Slip deformations may cause changes in the angles between the simulation cell vectors. In this case it leads to a decrease of $\gamma$ (Fig. 1d). The generation of defects increases the internal energy of the system, which explains the jump in the enthalpy in Figure 1f. A second plastic deformation (step 374) is a combined slip in $[0,0.3,0.47]$, which leads to a very high energy structure (Fig. 1f). The latter is therefore quickly (step 388) followed by another slip in $[0,0.3,0]$. The stacking fault is annealed in step 475 by a fourth slip [0,0.20,0.53]. The resulting structure after this first set of plastic deformations remains OEn. The total displacement vector is $[0,1.0,1.0]$. The 
small enthalpy difference between initial OEn and OEn after metastep 475 in Figure $1 \mathrm{f}$ is probably caused by potential energy cutoff effects due to the different shape of the respective simulation cells (cf. configurations 1 and 700 in Fig. 2). The simulation cell at this stage is constructed of following vectors with regard to the lattice vectors of OEn ( $\mathbf{a}_{\text {ortho }}, \mathbf{b}_{\text {ortho }}$ and $\left.\mathbf{c}_{\text {ortho }}\right): \mathbf{a}=\mathbf{a}_{\text {ortho }}+\mathbf{b}_{\text {ortho }}+\mathbf{c}_{\text {ortho }}, \mathbf{b}=2 \mathbf{b}_{\text {ortho }}$ and $\mathbf{c}=4 \mathbf{c}_{\text {ortho. }}$.

A second sequence of (100) stacking faults but in a different plane starts with step 712. Although the consecutive displacements in [010] look similar to those described above, related slips in [001] are quite different. The displacement in step 712 is [0,0.20,$0.16]$. It is followed by the following slips: $[0,0,0.68]$ in metastep $770,[0,0.32,-0.34]$ in metastep 847, [0,0.30,0.34] in metastep 872 and [0,0.18,0] in metastep 900. Displacive transitions between similar low-P and high-P phases lead to an additional displacement of $[0,0,-0.18]$. A low energy structure is reached again after a total displacement of one unit cell in [010] but only about 1/3[001] (metastep 900). Although the energy barrier for the first displacement of this sequence is similar to that of the previous one, consecutive slips only require very little additional energy. The enthalpy increase in metastep 865 is caused by the creating of an $\mathrm{Mg}$ point defect in the structure. This defect disappears again in metastep 1090 with release of energy, which is similar in magnitude to that of the creation. The point-defect free structure between metasteps 1090 and 1195 already has lower enthalpy than OEn. It seems to be of intermediate nature between OEn and high-P CEn (see Fig.2).

The third and final sequence of stacking fault displacements starts in metastep 1196, again in a different (100) plane. There are four slips with the following displacement vectors: [0,-0.20,-0.13] in metastep 1196, [0,-0.27,-0.39] in metastep 1200, [0,$0.34,0.40]$ in metastep 1250 and $[0,-0.18,0.48]$ in metastep 1292. The total slip of the sequence is one unit cell in [010] but in opposite direction, and again about 1/3[001]. The structure in metastep 1292 still has three Mg point defects, of which the first and second are annealed in metastep 1314. A defect free structure is eventually obtained after 1343 metasteps. It is interesting to note that the presence of defects seems to prevent a sudden drop in enthalpy at metastep 1314 in Figure 1e but leads to a rather smooth decrease of enthalpy. The final structure is identified as the high-P CEn phase after calculation and comparison of the diffraction patterns of high-P CEn in conventional setting and of the final structure of the metadynamics simulations. The 
latter is a supercell constructed by the following combination of lattice vectors of high-P CEn $\left(\mathbf{a}_{\text {clino }}, \mathbf{b}_{\text {clino }}, \mathbf{c}_{\text {clino }}\right): \mathbf{a}=2 \mathbf{a}_{\text {clino }}+\mathbf{b}_{\text {clino }}+\mathbf{c}_{\text {clino }}, \mathbf{b}=2 \mathbf{b}_{\text {clino }}$ and $\mathbf{c}=4 \mathbf{c}_{\text {clino. }}$.

\section{Discussion}

Metadynamics is expected to activate the slip deformations with the lowest activation energy. In all three deformation sequences slip occurs parallel to (100), which is plausible considering the strong Si-O bonds and consistent with experimental results (Coe and Kirby, 1975; Hugh-Jones et al., 1996). Since the intermediate structures within one sequence are high energy structures they could be expected to relax quickly towards lower energy structures. In this case, only the latter would be observable in the TEM. Hence, the transition would seem to follow the mechanism illustrated in Fig. 3. Since the deformation vector of the first slip sequence (a) to (b) contains only multiples of the unit cell vectors, plastic deformation without creation of additional stacking faults would be observed. The (b) to (c) and (c) to (d) deformations appear as (100)[001] slips, which is the assumed dominant slip system in orthopyroxenes. From the enthalpy increase during partial slip, an estimate of the stacking fault enthalpy can be calculated. For both OEn and high-P CEn, an enthalpy difference of $0.1 \mathrm{eV} / \mathrm{MgSiO}_{3}$ in Figure 1 corresponds to a stacking fault enthalpy of $38 \mathrm{meV} / \AA^{2}$, which is of the same order as the respective value for postperovskite at $120 \mathrm{GPa}$ (Oganov et al., 2005).

Experimental TEM studies of dislocation glide in OEn (van Duysen et al., 1985) show that both (100)[001] and (100)[010] slip systems may be activated, which is consistent with the present results. Dissociation of the dislocations into four partials was suggested with displacement vectors $\mathbf{R}_{1}=[0, \delta, \varepsilon], \mathbf{R}_{2}=[0, v, 0.5]$ and $\mathbf{R}_{3}=[0,-\delta-v, 0.5-\varepsilon]$. However, the parameters $\delta, \varepsilon$ and $v$ could not be determined from the diffraction contrast experiments. Using a simple model, a slip mechanism with $\delta=0, \varepsilon=1 / 3$ and $v=1 / 6$ was proposed (van Duysen et al., 1985). Our results confirm the TEM observations but suggest a different mechanism with $\delta=0.2, \varepsilon=0$ and $v=0.3$ in $\mathbf{R}_{1}$ and $\mathbf{R}_{2}$. The simulations suggest an additional partial dislocation $\mathbf{R}_{3}=[0,0.3,0]$ and a final $\mathbf{R}_{4}=[0,0.2,0.5]$. Since this new mechanism is obtained taking into account the energetics of the dislocation glide, it can be considered a possible low energy path for plastic deformation in OEn. Depending of the deformation conditions ( $P, T$, strain) other mechanisms are relevant, as well. In the present case, the two other slip deformations 
lead to the transformation of high-P CEn.

In conclusion, the atomistic modeling approach combining molecular dynamics simulation with metadynamics provides unprecedented insight into the plastic deformation behavior in pyroxenes. The knowledge of phase transformation and dislocation glide mechanisms in these minerals can contribute to a better understanding of the texture formation in relevant rocks. Further studies are needed at different $P-T$ conditions and for pyroxenes of different chemical composition.

\section{Acknowledgments}

The authors thank W. Müller, R. Abart, S. Speziale and two reviewers for helpful comments and discussions. R.M. was partially supported by Grant No. VEGA 1/2011/05 and the Centre of Excellence of the Slovak Academy of Sciences (CENG).

\section{References}

Aguado A, Bernasconi L, Jahn S, Madden PA (2003) Multipoles and interaction potentials in ionic materials from planewave-DFT calculations. Faraday Discuss 124:171-184

Ceriani C, Laio A, Fois E, Gamba A, Martoňák R, Parrinello M (2004) Molecular dynamics simulation of reconstructive phase transitions on anhydrous LiABW zeolite. Phys Rev B 70:113403

Coe RS, Kirby SH (1975) The orthoenstatite to clinoenstatite transformation by shearing and reversion by annealing: Mechanism and potential applications. Contrib Mineral Petrol 52:29-55

Coe RS, Müller WF (1973) Crystallographic orientation of clinoenstatite produced by deformation of orthoenstatite. Science 180:64-66

Hugh-Jones D, Sharp T, Angel R, Woodland A (1996) The transition of orthoferrosilite to high-pressure $\mathrm{C} 2 / \mathrm{c}$ clinoferrosilite at ambient temperature. Eur J Mineral 8:1337-1345

Jahn S (2007) High-pressure phase transitions in $\mathrm{MgSiO}_{3}$ orthoenstatite studied by atomistic computer simulation. Am Mineral (submitted)

Jahn S, Madden PA (2007) Modeling Earth materials from crustal to lower mantle conditions: A transferable set of interaction potentials for the CMAS system. Phys Earth Planet Int 162:129-139

Jahn S, Madden PA, Wilson M (2004) Dynamic simulation of the pressure-driven phase transformations in crystalline $\mathrm{Al}_{2} \mathrm{O}_{3}$. Phys Rev B 69:020106(R)

Kung J, Li B, Uchida T, Wang Y, Neuville D, Liebermann RC (2004) In situ measurements of the sound velocities and densities across the orthopyroxene $\rightarrow$ high-pressure clinopyroxene transition in $\mathrm{MgSiO}_{3}$ at high pressure. Phys Earth Planet Int 147:27-44

Madden PA, Heaton R, Aguado A, Jahn S (2006) From first-principles to material 
properties. J Mol Struc (Theochem) 771:9-18

Martoňák R, Donadio D, Oganov A, Parrinello M (2006) Crystal structure transformations in $\mathrm{SiO}_{2}$ from classical and ab initio metadynamics. Nat Mater 5:623-626

Martoňák R, Donadio D, Oganov AR, Parrinello M (2007a) From 4- to 6coordinated silica: transformation pathways from metadynamics. Phys Rev B 76:014120

Martoňák R, Laio A, Bernasconi M, Ceriani C, Raiteri P, Zipoli F, Parrinello M (2005) Simulation of structural phase transitions by metadynamics. Z Kristall 220:489-498

Martoňák R, Laio A, Parrinello M (2003) Predicting crystal structures: The Parrinello-Rahman method revisited. Phys Rev Lett 90:075503

Martoňák R, Oganov AR, Glass CW (2007b) Crystal structure prediction and simulations of structural transformations: metadynamics and evolutionary algorithms. Phase Transit 80:277-298

Martyna GJ, Tobias DJ, Klein ML (1994) Constant pressure molecular dynamics algorithms. J Chem Phys 101:4177-4189

McLaren AC, Etheridge MA (1976) A transmission electron microscopy study of naturally deformed orthopyroxene. I. Slip mechanisms. Contrib Mineral Petrol 57:163-177

Miyake A, Shimobayashi N, Miura E, Kitamura M (2002) Molecular dynamics simulations of phase transition between high-temperature and high-pressure clinoenstatite. Phys Earth Planet Int 129:1-11

Oganov AR, Martoňák R, Laio A, Raiteri P, Parrinello M (2005) Anisotropy of Earth's D" layer and stacking faults in the $\mathrm{MgSiO}_{3}$ post-perovskite phase. Nature 438:1142-1144

Presnall DC (1995) Phase diagrams of Earth-forming minerals. In: Mineral Physics and Crystallography, A Handbook of Physical Constants. Vol. 2 of AGU Reference Shelf. American Geophysical Union, pp 248-268

Sasaki S, Takeuchi Y, Fujino K, Akimoto S (1982) Electron-density distributions of three orthopyroxenes, $\mathrm{Mg}_{2} \mathrm{Si}_{2} \mathrm{O}_{6}, \mathrm{Co}_{2} \mathrm{Si}_{2} \mathrm{O}_{6}$, and $\mathrm{Fe}_{2} \mathrm{Si}_{2} \mathrm{O}_{6} . \mathrm{Z}$ Kristall 156:279-297

Shimobayashi N, Miyake A, Kitamura M, Miura E (2001) Molecular dynamics simulations of the phase transition between low-temperature and hightemperature clinoenstatites. Phys Chem Minerals 28:591-599

Thompson RM, Downs RT (2003) Model pyroxenes I: Ideal pyroxene topologies. Am Mineral 88:653-666

van Duysen JC, Doukhan N, Doukhan JC (1985) Transmission electron microscope study of dislocations in orthopyroxene $(\mathrm{Mg}, \mathrm{Fe})_{2} \mathrm{Si}_{2} \mathrm{O}_{6}$. Phys Chem Minerals $12: 39-44$

Weidner DJ, Wang H, Ito J (1978) Elasticity of orthoenstatite. Phys Earth Planet Int 17:P7-P13

Woodland AB (1998) The orthorhombic to high-P monoclinic phase transition in $\mathrm{Mg}-\mathrm{Fe}$ pyroxenes: Can it produce a seismic discontinuity? Geophys Res Lett 25:1241-1244 
Woodland AB, Angel RJ (1997) Reversal of the orthoferrosilite - high-P clinoferrosilite transition, a phase diagram for $\mathrm{FeSiO}_{3}$ and implications for the mineralogy of the Earth's upper mantle. Eur J Mineral 9:245-254 


\section{Tables}

Table 1: Lattice parameters $(\AA)$ and elastic constants (GPa) of OEn at ambient conditions predicted by the AIM potential and compared to experimental data of Sasaki et al. (1982) and Weidner et al. (1978)

\begin{tabular}{|c|c|c|}
\hline & AIM & Experiment \\
\hline$a_{\text {ortho }}$ & 17.93 & 18.23 \\
$b_{\text {ortho }}$ & 8.81 & 8.82 \\
$c_{\text {ortho }}$ & 5.16 & 5.18 \\
\hline $\mathrm{c}_{11}$ & 218 & 224 \\
$\mathrm{c}_{22}$ & 139 & 178 \\
$\mathrm{c}_{33}$ & 216 & 214 \\
$\mathrm{c}_{44}$ & 83 & 78 \\
$\mathrm{c}_{55}$ & 65 & 76 \\
$\mathrm{c}_{66}$ & 69 & 82 \\
$\mathrm{c}_{12}$ & 87 & 72 \\
$\mathrm{c}_{13}$ & 63 & 54 \\
$\mathrm{c}_{23}$ & 56 & 53 \\
\hline
\end{tabular}

\section{Figure captions}

Fig. 1: Evolution of simulation cell lengths, cell angles and enthalpy per formula unit starting from the OEn phase during metadynamics at $P=15 \mathrm{GPa}, T=1000 \mathrm{~K}$ (a), (c), (e) and after relaxing the configurations to hydrostatic conditions at $P=15 \mathrm{GPa}, T=300 \mathrm{~K}$ (b), (d) and (f), respectively.

Fig. 2: Selected relaxed structures from metadynamics simulation in projections along [010] (above) and [001] (below). The $\mathrm{SiO}_{4}$ tetrahedra are represented by polyhedra and the $\mathrm{Mg}$ cations by spheres. $a^{*}$ is the projection of $a$ perpendicular to $b$ and $c$. The position of the (100) slip planes and the respective slip directions are indicated by arrows.

Fig. 3: Schematic representation of the transformation mechanism projected along (010): (a) original OEn, (b) OEn after metastep 475, (c) intermediate phase at metastep 1150 and (d) final high-P CEn. Circles represent the Mg layers (cf. Fig. 2). The [001] displacement of the $\mathrm{SiO}_{4}$ chains is represented by triangles. The (100) slip planes for the three different deformation sequences are indicated by horizontal lines. The respective arrows show the directions of slip. Also indicated is the change of the simulation cell due to the deformation. In this projection, the most prominent change is observed in the simulation cell angle $\beta$. 

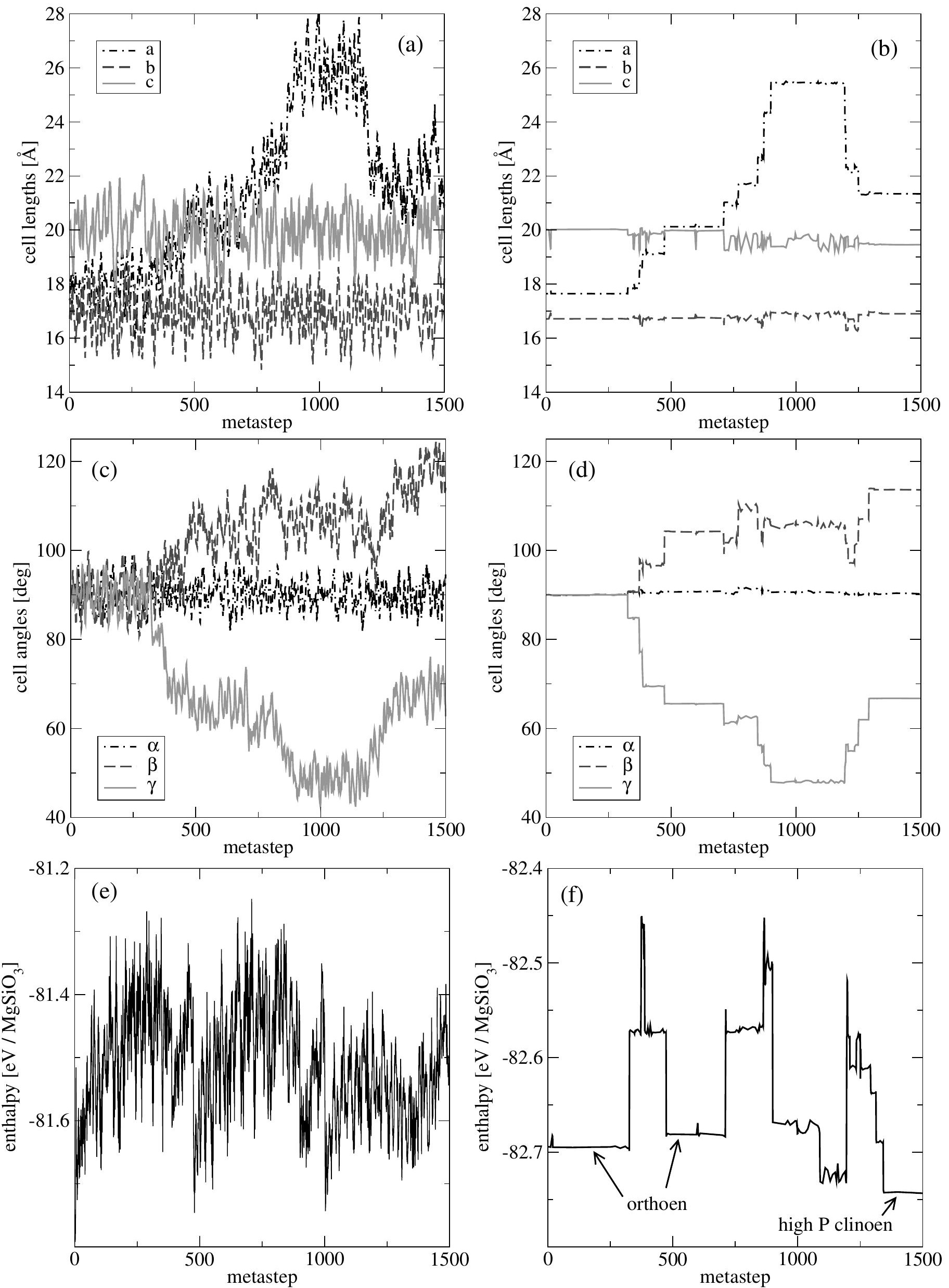
metastep 1

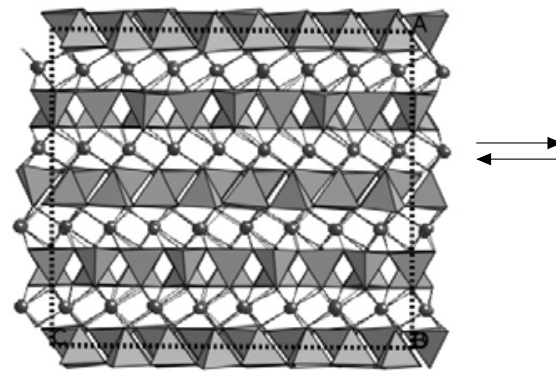

700
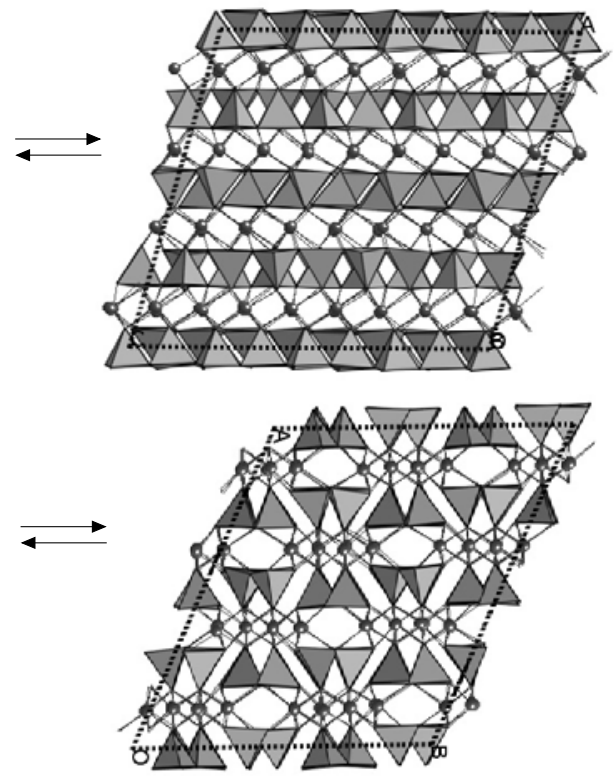

1150
800
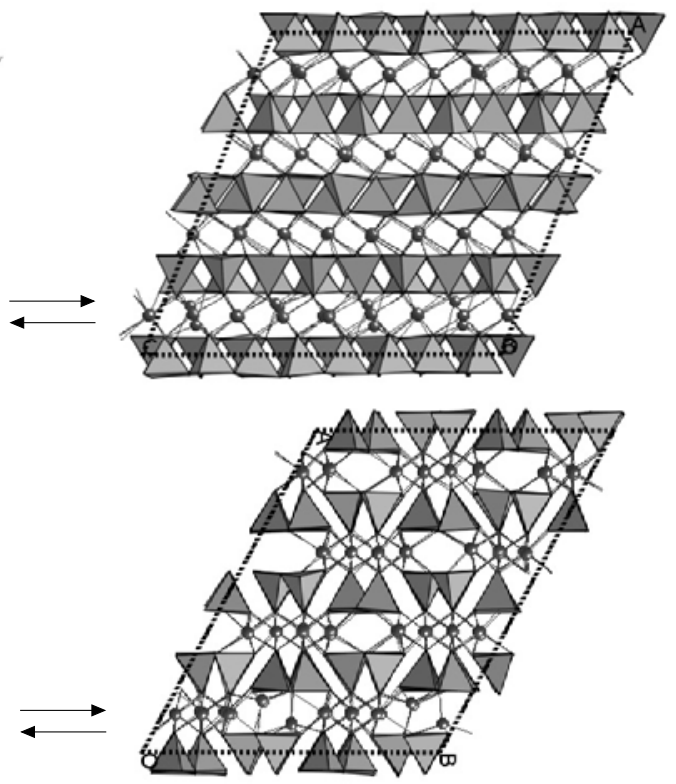

1400
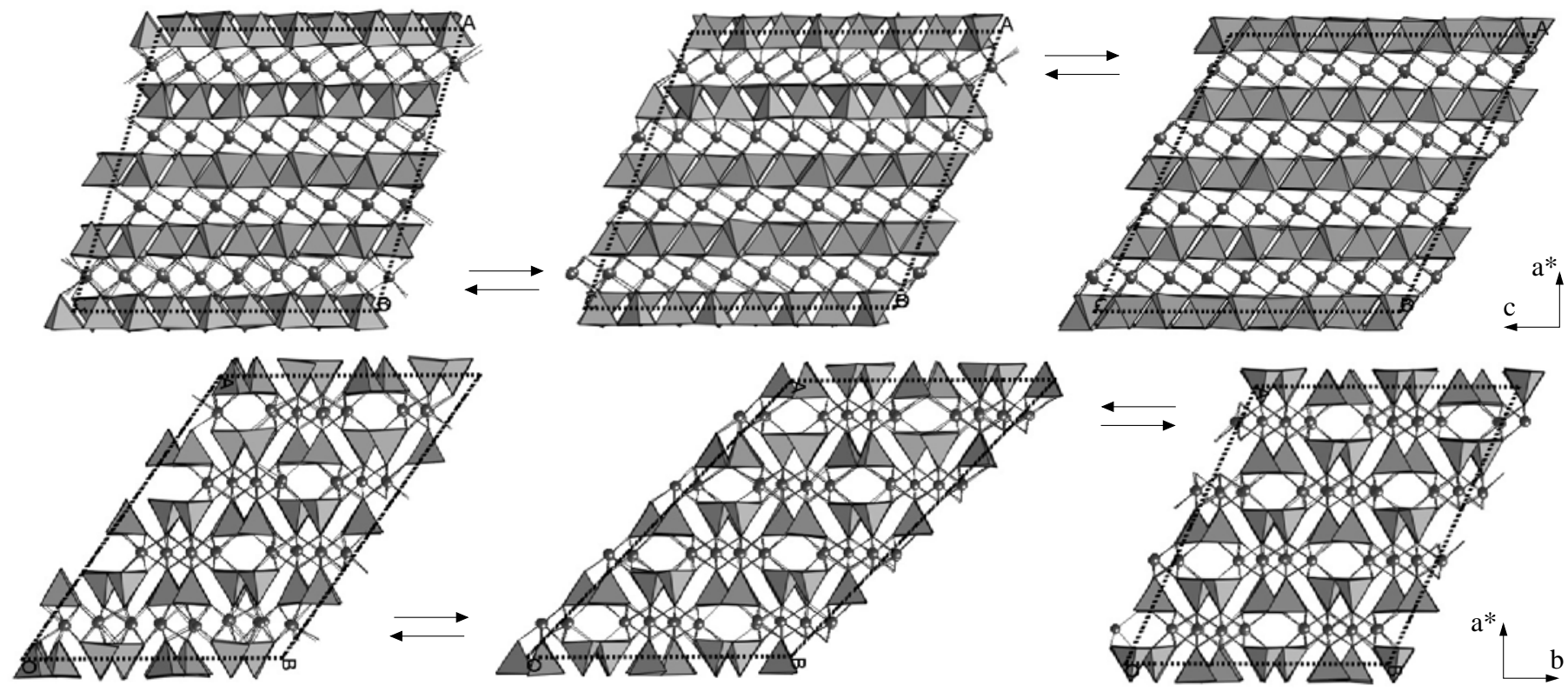
